\title{
Classification of Supernovae
}

\author{
Massimo Turatto \\ Osservatorio Astronomico di Padova, vicolo dell'Osservatorio 5, I-35122, Padova, Italy
}

\begin{abstract}
The current classification scheme for supernovae is presented. The main observational features of the supernova types are described and the physical implications briefly addressed. Differences between the homogeneous thermonuclear type Ia and similarities among the heterogeneous core collapse type Ib, Ic and II are highlighted. Transforming type IIb, narrow line type IIn, supernovae associated with GRBs and few peculiar objects are also discussed.
\end{abstract}

\section{Introduction}

An adequate and satisfactory taxonomy for a class of objects should fulfill certain elementary characteristics such as mutual exclusion, exhaustiveness, nonambiguity, repeatability and usefulness. The taxonomy of supernovae ( $\mathrm{SNe}$ ) has been progressively developing since 1941 when Minkowskii 88 first recognized that at least two main types of SNe exist. Since then several new types of SNe have been introduced, some have been dismissed, and others have held their ground. After an early "Linnaeus stage" during which the classification was based on the recognition of the observational characteristics, the concept of SN type has been refined to include "genetic" information. Nevertheless the present day classification scheme is still not satisfactory. In particular, it is ambiguous and non-exhaustive (many objects are still classified as "peculiar") and the nomenclature of the taxonomic groups, determined by historical reasons, is confusing. However, since classification is a process which mankind naturally and instinctively carries out in order to sort out and understand vast arrays, the available SN classification scheme remains extremely important and useful.

Extensive reviews on the SN taxonomy can be found in [4462 134]. Here we give a brief update on the subject. More details can be found in other chapters in this volume.

\section{Main Supernova Types}

The classification of SNe is generally performed on the optical spectra but, to some extent, also on their light curves. Since SNe are brighter near their maximum light, for obvious reasons the classification is based on the early spectra, which consist of a thermal continuum and P-Cygni profiles of lines formed by resonant scattering. This means that the SN types are assigned on the basis of the chemical and physical properties of the outermost layers of the exploding stars. 


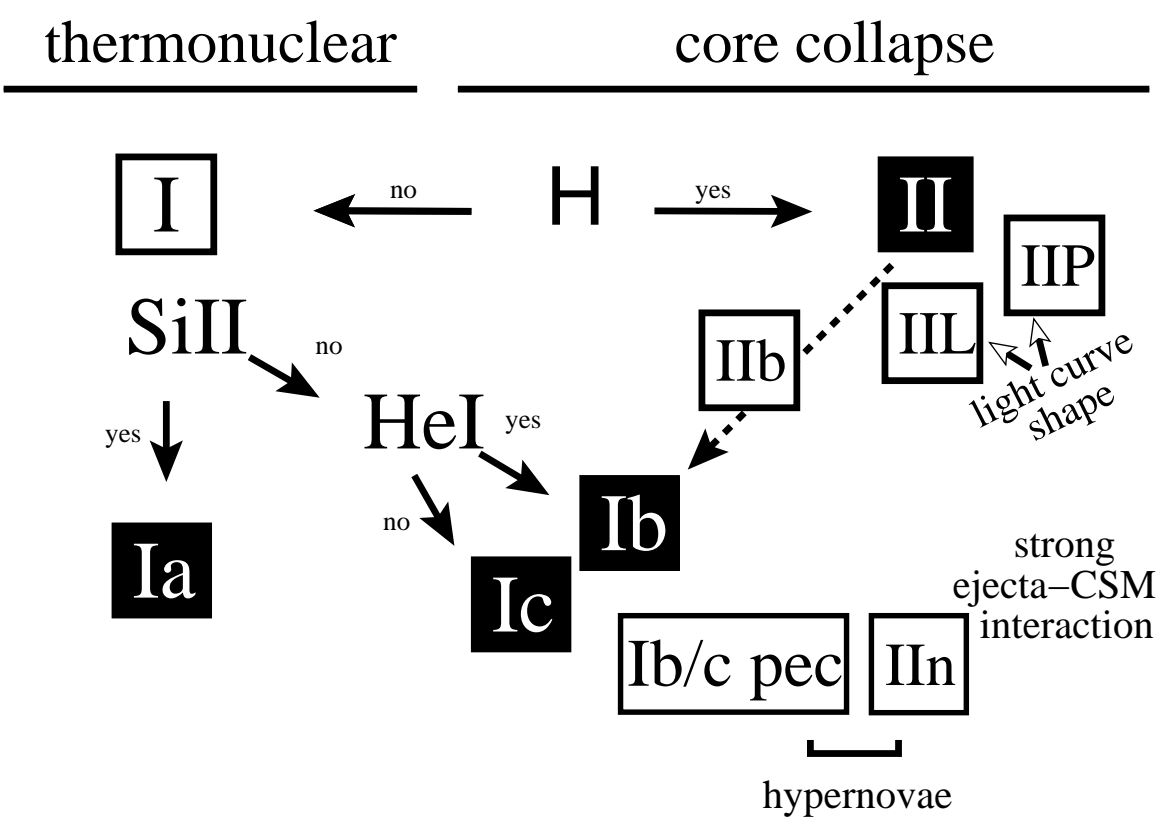

Fig. 1. The current classification scheme of supernovae. Type Ia SNe are associated with the thermonuclear explosion of accreting white dwarfs. Other SN types are associated with the core collapse of massive stars. Some type Ib/c and IIn SNe with explosion energies $E>10^{52} \mathrm{erg}$ are often called hypernovae.

Only in recent years have late time observations contributed to differentiating various subtypes.

The first two main classes of SNe were identified [88] on the basis of the presence or absence of hydrogen lines in their spectra: SNe of type I (SNI) did not show $\mathrm{H}$ lines, while those with the obvious presence of $\mathrm{H}$ lines were called type II (SNII). Type I SNe were also characterized by a deep absorption at 6150 $\AA$ which was not present in the spectra of some objects, therefore considered peculiar 1617. In 1965, Zwicky 143 introduced a schema of five classes but in recent years the scarcely populated types III, IV and V have been generally included among type II SNe.

In the mid-1980s, evidence began to accumulate that the peculiar SNI formed a class physically distinct from the others. The objects of the new class, characterized by the presence of HeI [5863], were called type Ib (SNIb), and "classical" SNI were renamed as type Ia (SNIa). The new class further branched into another variety, SNIc, based on the absence of He I lines. Whether these are physically distinct types of objects has been long debated 62 135]. In several contexts they are referred to as $\mathrm{SNIb} / \mathrm{c}$. 


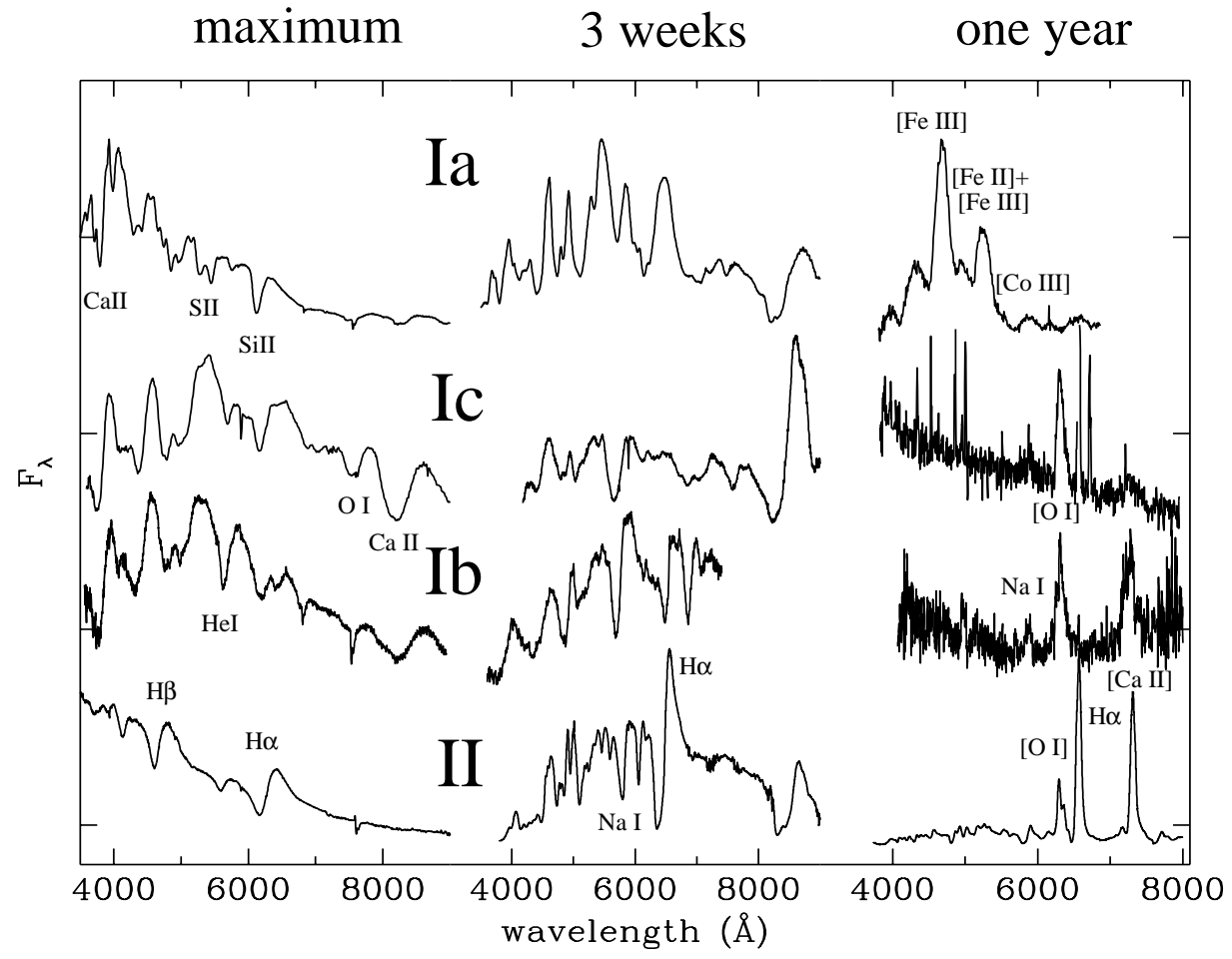

Fig. 2. The spectra of the main SN types at maximum, three weeks, and one year after maximum. The representative spectra are those of SN1996X for type Ia 109], of SN1994I (left and center) 35] and SN1997B (right) for type Ic, of SN1999dn (left and center) and SN1990I (right) for type Ib, and of SN1987A 99] for type II. At late times (especially in the case of the type Ic SN1997B) the contamination from the host galaxy is evident as an underlying continuum plus unresolved emission lines. In all figures of this paper the spectra have been transformed to the parent galaxy rest frame.

\section{Type Ia Supernovae}

Type Ia SNe have become very popular in the last decade because of their role in determining the geometry of the Universe with their high luminosity and relatively small luminosity dispersion at maximum. An extensive review of the properties of SNIa has recently appeared [69].

SNIa are discovered in all types of galaxies, also in ellipticals [8], and are not associated with the arms of spirals as strongly as other SN types 85128. The spectra are characterized by lines of intermediate mass elements such as calcium, oxygen, silicon and sulfur during the peak phase and by the absence of $\mathrm{H}$ at any time (see, e.g., Fig. (2). With age the contribution of the Fe lines increases and several months past maximum the spectra are dominated by [Fe II] and [Fe III] lines. The overall homogeneous spectroscopic and photometric behavior has led 
to a general consensus that they are associated with the thermonuclear explosion of a white dwarf [20].

Nevertheless, during the past decade early suggestions of significant differences among SNIa 9102] have been confirmed by new, high signal-to-noise data. The crucial year was 1991, when the bright, slowly declining SN1991T [74105, and the faint, intrinsically red and fast declining SN1991bg [50 71119] were discovered. Other under- and over-luminous objects have been found since then [66.

The analysis of homogeneous sets of optical data led to the discovery of a correlation between the peak luminosity and the shape of the early light curve with brighter objects having a slower rate of decline than dimmer ones 9798100104 . This correlation has been employed in restoring SNIa as useful distance indicators up to cosmological distances. A correlation between the photometric and the spectroscopic properties was also found 90 .

It is known that the peak luminosity of SNIa is directly linked to the amount of radioactive ${ }^{56} \mathrm{Ni}$ produced in the explosion 35. Hence SNIa, having different magnitudes at maximum, are probably the result of the synthesis of different amounts of radioactive ${ }^{56} \mathrm{Ni}$. Moreover, there are indications of large variances (up to a factor 2) in the total mass of the ejecta [24.

Observations at other wavelengths have provided very useful information. In particular, infrared and I-band light curves have shown that the light curves of SNIa are characterized by a secondary peak 20-30 days after the B maximum 397486115. Remarkable exceptions are the faint objects like SN1991bg.

The afore mentioned diversity of SNIa persists up to very late epochs. The light curves of faint objects are steeper than those of other SNIa, probably because of a progressive transparency to positrons from radioactive decay 2487. Faint SNIa also show slower expansion velocity of the emitting gas both at early 43 125] and late epochs 82.

The calibration of the absolute magnitudes of a number of type Ia $\mathrm{SNe}$ by Cepheids has provided the zero point for determining the Hubble Constant $\left(\mathrm{H}_{0}\right)$. Average values based on limited samples and different recipes range from $\mathrm{M}_{V}=-19.34$ to $\mathrm{M}_{V}=-19.64$ with small dispersions 6068107116. The recent determination of the Cepheid distance to the host galaxy of SN1991T seems to confirm that it is brighter $\left(\mathrm{M}_{V}=-19.85 \pm 0.29\right)$ than the spectroscopically normal supernovae [108].

SN1991T was also the first SNIa to show photometric and spectroscopic evidence of a light echo from circumstellar dust [11]. So far the only other SNIa to show the same phenomenon was the slowly declining, spectroscopically normal SN1998bu [27. In addition to the light echo, these two SNe suffered strong reddening, quite unusual among known, standard SNIa, suggesting that slow decliners may be associated to younger population objects. The fact that, on average, SNIa in late type galaxies have slower decline rates (hence are more luminous) than SNIa in early type galaxies had been already suggested [254361126]. 
One current issue important for the application of SNIa to cosmology is whether they evolve with redshift. Indeed, the lower metallicity of the progenitors at higher redshift might result in systematic differences in brightness.

\section{Type Ib and Ic Supernovae}

Type Ib and Ic appear only in spiral type galaxies 8101 and have been associated with a parent population of massive stars, perhaps more massive than SNII progenitors 128. SNIb/c exhibit relatively strong radio emission with steep spectral indices and fast turn-on/turn-off [132, which is thought to arise from the SN shock interaction with a dense circumstellar medium [2829]. They are, therefore, usually thought to be associated with the core collapse of massive stars which have been stripped of their outer $\mathrm{H}$ (and possibly $\mathrm{He}$ ) envelope.

The introduction of this class of SNe is recent. As mentioned above, they were classified with other SNI until the mid-1980s, when late time observations of SNe 1983N, 1984L and 1985F highlighted the physical differences from SNIa 31475863138 . The characterizing features are the absence of $\mathrm{H}$ and Si II lines and the presence of He I. The excited levels of He producing such lines are thought to be populated by fast electrons accelerated by $\gamma$-rays from the decay of ${ }^{56} \mathrm{Ni}$ and ${ }^{56} \mathrm{Co} 6375$. It was soon recognized that some objects did not show strong He lines [136] and the class of helium poor type Ic was proposed [62 135].

In order to investigate the physical differences between these two classes, the signatures of He were searched carefully. The He I $\lambda 10830$ line in Type Ic was first found in the spectra of SN1994I 49] with velocity as high as $17,000 \mathrm{~km} \mathrm{~s}^{-1}$ 35. It was noted that even as little as $0.1 \mathrm{M}_{\odot}$ of helium at such high expansion velocities implies an energy of about $3 \times 10^{51} \mathrm{erg}$ in the outer shell alone. Unless very high explosion energies are involved, the amount of He has to, therefore, be much smaller 35. Other SNIc have shown He lines at similar (SNe 1987M and 1988L) and slower (SN1990B [38]) velocities. Thus the presence of some amount of He seems to be common to several SNIc progenitors.

Helium has been unambiguously identified also in the spectra of the recent type Ic SN1999cq [76. These lines have expansion velocities much lower than other lines, indicating that the ejecta interacts with a dense shell of almost pure He originating from a stellar wind or mass transfer to a companion. These findings support the idea that SNIc differ from SNIb by the He abundance rather than by the amount of mixing of ${ }^{56} \mathrm{Ni}$ in the helium envelope.

Absorption features attributed to $\mathrm{H} \alpha$ were first identified in the spectra of the type Ib SNe 1983N and 1984L 137. Recent analysis of the spectra of 11 objects has suggested that detached $\mathrm{H}$ is generally present in SNIb 22 . The optical depths of $\mathrm{H}$ and $\mathrm{He}$ are not very high, so that modest differences in the He I line optical depths might transform type Ib into type Ic objects.

In addition to the different strengths of He lines, it has been suggested 76 that permitted oxygen lines are relatively stronger in type Ic than in Ib and the nebular emission lines broader. In general, type Ib appear more homogeneous than type Ic. 


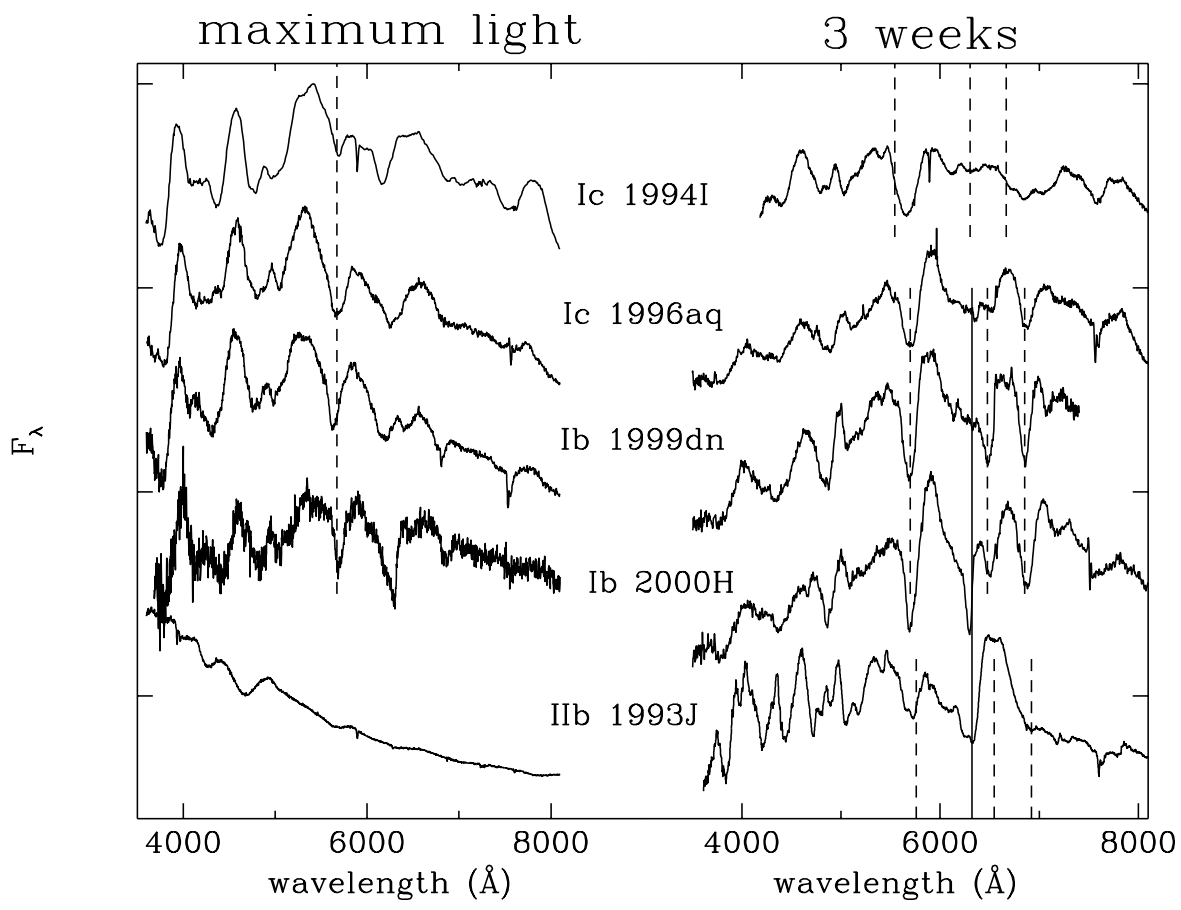

Fig. 3. Comparison among the spectra of SNe reported as type $\mathrm{Ib} / \mathrm{c}$ in the Asiago SN Catalogue [8] (left: at maximum light; right: 3 weeks later). In addition to the spectra of SN1994I (Ic) and 1999dn (Ib) shown in Fig. 2 the spectra of SN1996aq (Ic), 2000H (Ib) 22 and 1993J (IIb) 7] are displayed. With the exception of SN1993J, the spectra at maximum are rather similar. For reference the position of He I $\lambda 5876$ (blueshifted by $10,500 \mathrm{~km} \mathrm{~s}^{-1}$ ) is marked with a dashed vertical line. The dashed lines on the right indicate the positions of He I $\lambda \lambda$ 5876, 6678 and 7065 (blueshifted by $16,900 \mathrm{~km} \mathrm{~s}^{-1}$ for 1994I, 6,000 $\mathrm{km} \mathrm{s}^{-1}$ for SN1993J and 9,000 $\mathrm{km} \mathrm{s}^{-1}$ for other objects). $\mathrm{H} \alpha$ (solid line, blueshifted by $11,000 \mathrm{~km} \mathrm{~s}^{-1}$ ) is also shown. While He I lines in SN1994I are detectable only through a detailed analysis, they are prominent in the spectra of SN1996aq, which should be reclassified as a SNIb.

The light curves of SNIb/c have been divided in two groups depending on the luminosity decline rate 34]. However, the suggestion that type Ic include both fast and slow decliners while Ib seem to prefer slow declines has been challenged by the existence of SNIb with fast light curves, e.g., SNe 1990I and 1991D.

With this variety, the classification of new objects based on a single spectrum is difficult, and to some extent subjective, so that more detailed analyses of the objects often reveal discrepancies from early classifications. This is illustrated in Fig. 3 which presnets the optical spectra of a number of objects reported as type $\mathrm{Ib} / \mathrm{c}$ in the Asiago SN Catalog 8 . 


\subsection{Type IIb}

A few objects have been found to have early time spectra similar to type II (i.e. with prominent $\mathrm{H}$ lines) and late time spectra similar to type $\mathrm{Ib} / \mathrm{c}$ SNe. For this reason they have been called type IIb. The first SNIIb was SN1987M [42, but the best studied example, and one of the best studied SNe ever, was SN1993J in M81. The analysis of seven years of observations of this object has been recently published [77/79], and will be discussed in more detail in other chapters of this volume. More examples of transition objects are SN1996cb [141] and probably SN1997dd 78.

Fig. [3] shows that while the early spectrum of SN1993J was almost featureless with a blue continuum and broad $\mathrm{H}$ and He I $\lambda 5876$ lines typical of SNII, already three weeks later it displayed progressively stronger He I $\lambda \lambda$ 5876, 6678 and 7065 lines characteristic of SNIb.

The light curve of SN1993J was unusual with a narrow peak followed by a secondary maximum, recalling the behavior of SN1987A if the time axis were reduced by a factor of four. After another rapid luminosity decline around 50 days past the explosion, the light curve settled into an almost exponential tail with a decline rate faster than normal SNII and similar to that of SNIa, indicative of a small mass for the ejecta.

Indeed, the photometry of the progenitor of SN1993J taken before the explosion is inconsistent with the spectral energy distribution of a single star, but requires the composition of a K0Ia spectrum with a hot component [1. The radio and X-ray emission of SN1993J 11129142 have been attributed to circumstellar interaction [54. Another indication comes from the boxy shape of the emission lines of late time optical spectra 7794. Circumstellar gas in proximity to the exploding star was also revealed through the detection of narrow coronal lines persisting for a few days after the explosion [13.

These SNe transforming from type II to Ib/c constitute the previously missing link between envelope retaining and envelope stripped SNe.

\section{Type II Supernovae}

Type II SNe are characterized by the obvious presence of $\mathrm{H}$ in their spectra. They avoid early type galaxies [ $\underline{8}$, are strongly associated with regions of recent star formation [81128] and are commonly associated with the core collapse of massive stars 140.

SNII display a wide variety of properties both in their light curves 9293 . and in their spectra 44. Four subclasses of SNII are commonly mentioned in the literature: IIP, IIL, and IIn in addition to the above mentioned IIb. However, a number of peculiar objects do not fit into any of these categories.

SNIIP (Plateau) and SNIIL (Linear) constitute the bulk of all SNII, and are often referred as normal SNII. The subclassification is made according to the shape of the optical light curves [10. The luminosity of SNIIP stops declining shortly after maximum forming a plateau 2-3 months long during which a 
recombination wave moves through the massive hydrogen envelope releasing its internal energy. SNIIL, on the other hand, show a linear, uninterrupted luminosity decline, probably because of a lower mass envelope. Indeed the two classes are not separated and there are a number of intermediate cases with short plateaus, e.g., SN1992H [36]. A quantitative criterion for the classification of the light curves has been proposed on the basis of the average decline rate of the first 100 days 93. Starting 150 days past maximum the luminosity of both types settles into an exponential decline, consistent with complete (or constant) trapping of the energy release of the radioactive decay of ${ }^{56} \mathrm{Co}$ into ${ }^{56} \mathrm{Fe}$.

No major spectral differences exist between SNIIP and SNIIL, although there are recurrent claims that SNIIL do not show the blueshifted absorption of the P-Cygni profile evident in normal SNIIP. Indeed, a statistical analysis has shown that the presence or absence of the P-Cygni absorption is correlated with the absolute brightness at maximum 93 .

The progenitors of SNIIL are believed to have $\mathrm{H}$ envelopes of the order of 1-2 $\mathrm{M}_{\odot}$, much smaller than those of SNIIP (typically $10 \mathrm{M}_{\odot}$ ) probably due to mass loss during the progenitor evolution. A general scenario has been proposed in which common envelope evolution in massive binary systems with varying mass ratios and separations of the components can lead to various degrees of stripping of the envelope [89]. According to this scenario the sequence of types IIP-IILIIb-Ib-Ic in Fig. 1 is ordered according to a decreasing mass of the envelope.

SNIIL are often radio sources [132] and show UV excess attributed to Compton scattering of photospheric radiation by high speed electrons in shock-heated circumstellar medium (CSM) material 52 53. A number of SNIIL, e.g., SNe 1994aj and 1996L 1214, have shown lines with double P-Cygni profiles (sometimes dubbed SNIId, "d" for double) indicating the presence of strong wind episodes shortly before the explosion. These and other SNIIL show a flattening in the light curves at late stages, a broadening of the spectral lines, and $\mathrm{H} \alpha$ fluxes greater than those expected from purely radioactive models 234170124 . These features have been interpreted as signatures of the onset of interaction between the ejecta and the circumstellar material.

The epochal SN1987A in the Large Magellanic Cloud (LMC), the first SN to be observed by naked eye in the last four centuries, was a "not very peculiar" type II SN with a plateau. Extensive observations at all wavelengths have explored the nature of this object in detail (see, e.g., 4 and references therein). The detection of neutrinos from SN1987A has been a spectacular confirmation of the theory of core collapse 1864. The contribution of this object to our understanding of supernovae is addressed by McCray in another chapter in this volume.

Because of its closeness, SN1987A was the first SN for which it has been possible to unambiguously identify the progenitor, the B3 I star Sk-69 202. More recent, high spatial resolution prediscovery images of nearby galaxies are providing new insights into the nature of the precursor stars of other core collapse SNe. In addition to the case of SN1993J in M81 mentioned above, tight constraints on the mass of the progenitors of SNe 1999em and 1999gi have been published $\left(M(1999 \mathrm{gi})<9_{-2}^{+3} \quad \mathrm{M}_{\odot}\right.$ and $M(1999 \mathrm{em})<12 \pm 1 \quad \mathrm{M}_{\odot}$ [113114] $)$. 
SN1987A was typical in both the explosion energy $\left(\sim 10^{51} \mathrm{erg}\right)$ and the amount of ejected radioactive material $\left(\mathrm{M}\left({ }^{56} \mathrm{Ni}\right)=0.07 \mathrm{M}_{\odot}\right)$. Recent observations of other SNe seem to indicate a considerable dispersion around such values. High explosion energies are required to explain the high luminosities and kinetic energies of hypernovae (see, e.g., (6) and a large mass of radioactive ${ }^{56} \mathrm{Ni}(0.3$ $\left.\mathrm{M}_{\odot}\right)$ has been measured for SN1992am [112. At the other extreme the faint SN1997D had a considerably smaller explosion energy $\left(\sim\right.$ few $\left.\times 10^{50} \mathrm{erg}\right)$ and returned to the ISM a mass of radioactive material as small as $M\left({ }^{56} \mathrm{Ni}\right)=0.002$ $\mathrm{M}_{\odot}[122$. The discovery of similar objects (e.g., SN1999eu) indicates that faint, under energetic SNII may represent a non-negligible fraction of all core collapse SNe. These objects are the best candidates for the detection of signatures of black hole formation and for providing support to the black hole-forming SN scenario 615].

\subsection{Type IIn}

A number of peculiar SNII have been grouped into the class of SNIIn ("n" denoting narrow emission lines [110]). The spectra of these objects have a slow evolution and are dominated by strong Balmer emission lines without the characteristic broad absorptions. The early time continua are very blue, He I emission is often present and, in some cases, narrow Balmer and $\mathrm{Na}$ I absorptions are visible corresponding to expansion velocities of about $1,000 \mathrm{~km} \mathrm{~s}^{-1}$ [91, reminiscent of SNIId (see 45). Unresolved forbidden lines of [OI], [OIII], and of highly ionized elements such as [FeVII], [FeX], and $[\mathrm{AX}]$ are sometimes present.

One of the best cases is SN1988Z, which has been observed in the optical, radio and X-rays for over ten years [2]. Balmer lines, in particular $\mathrm{H} \alpha$, show a well defined multiple component structure with broad (FWHM $\sim 15,000 \mathrm{~km} \mathrm{~s}^{-1}$ ), intermediate $\left(2,200 \mathrm{~km} \mathrm{~s}^{-1}\right)$ and narrow $\left(<700 \mathrm{~km} \mathrm{~s}^{-1}\right)$ components with different time evolution [121. SN1995N was a remarkably similar object, detected in radio and X-rays 51132 .

It is commonly believed that what we observe in these objects is the result of interaction between the ejecta and a dense CSM, which transforms the mechanical energy of the ejecta into radiation 32117. The interaction of the fast ejecta with the slowly expanding CSM generates a forward shock in the CSM and a reverse shock in the ejecta. The shocked material emits energetic radiation whose characteristics strongly depend on the density of both the CSM and the ejecta, and on the properties of the shock [30. Thus the great diversity of observed SNIIn can provide clues to the different history of the mass-loss in the late evolution of progenitors.

A systematic search for radio emission from SNIIn has produced contradictory results [130. This might be due to the high degree of heterogeneity and to a bias in the classification of the targets. In fact, a solid classification criterion for these SNe is still missing, and often objects classified as SNIIn turn out to be normal when they undergo closer scrutiny (e.g., SN1989C [120]).

A remarkable SNIIn is the recent SN1998S. Contrary to most other SNIIn, the spectrum evolved rapidly and seems to be the result of the interaction between 


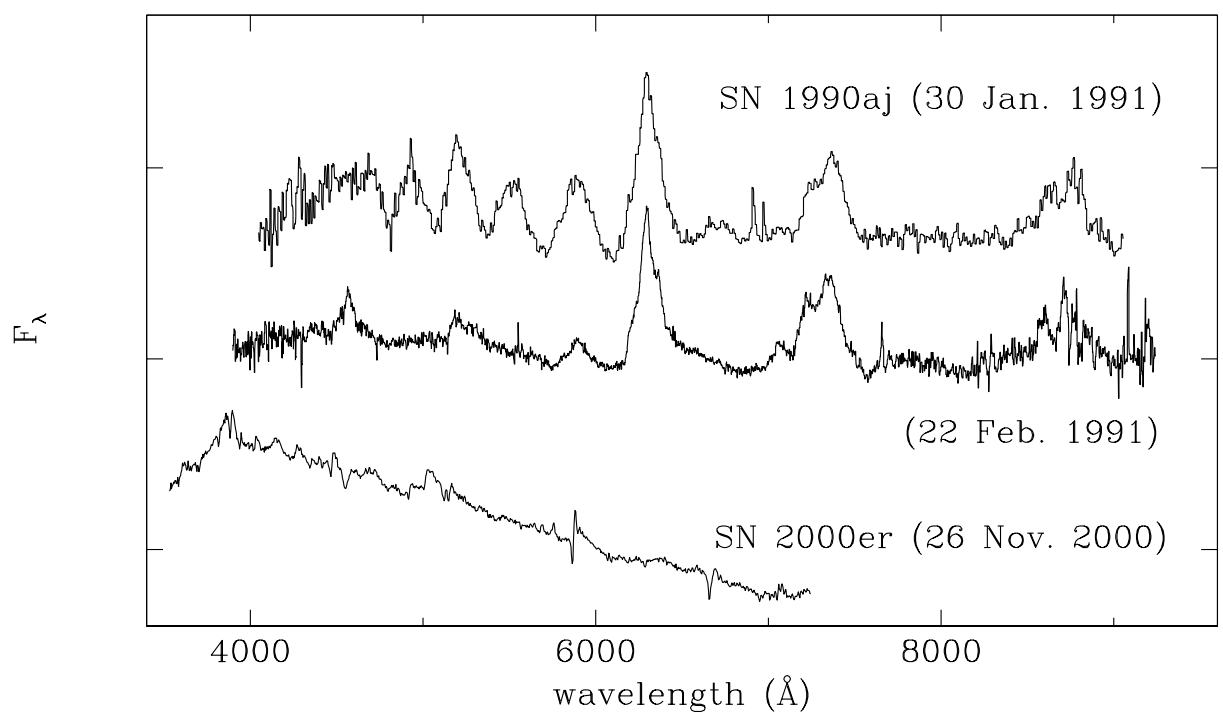

Fig. 4. Spectra of SN1990aj, a SNIb/c with exceptionally strong Fe lines at late epochs, and SN2000er, a bright, fast declining SN with evidence of narrow He I lines in the spectra at maximum.

the supernova and a two-component progenitor wind [40. Polarimetric studies indicate significant asphericity for its continuum-scattering environment [3]. The early spectra are well modeled by lines arising primarily in the circumstellar region while later spectra are dominated by the supernova ejecta 72 .

\section{$6 \quad \mathrm{SNe}$ and GRBs}

Other chapters in this volume discuss the association of SN1998bw with GRB980425 and the possibility that at least some GRBs originate from SN explosions.

In addition to its stringent spatial and temporal association to GRB980425 5567, SN1998bw was peculiar in many respects. The spectrum at early phases was unprecedented and led to different classifications (Ib [106] or peculiar Ic 46 95]). SN1998bw was as bright as an SNIa and displayed expansion velocities as high as $3 \times 10^{4} \mathrm{~km} \mathrm{~s}^{-1}$, suggesting that it was the result of an extremely energetic explosion ( $>10^{52} \mathrm{erg}$ ), even if different degrees of asymmetry and beaming allow for a broad range of values 6567139. Its very powerful radio emission has been interpreted as due to the presence of a mildly relativistic blast wave interacting with a clumpy, structured CSM deriving from a complex mass-loss history (see the chapter by Weiler et al. in this volume and [133]). The complete dataset of optical and IR observations spanning over one year after the explosion has recently appeared [96. The analysis of the late time spectra 84 seems to confirm that the explosion was asymmetric as suggested by polarimetry 
65. The presence of underlying SNe similar to SN1998bw has been invoked to explain the anomalous rebrightening in light curves of other GRB afterglows at high redshift 19103 .

Other known SNe, SN1997ef [57] and SN1998ey [56], bear some spectroscopic resemblance to SN1998bw. In particular, SN1997ef was possibly associated with GRB971115 131] and, although it was fainter than SN1998bw, its kinetic energy was close to that of SN1998bw [83. The case of the bright SNIc 1992ar [37, which occurred at about two $\sigma$ from the position of GRB920616 139, is also very interesting.

Additional SNe possibly associated with GRBs are SN1997cy, (GRB970514 [59 139]), observationally classified as SNIIn and possibly the brightest SN ever observed [123], and its twin SN1999E 26] (GRB980910 [118). As in the case of the type IIn SN1988Z, they show evidence for strong ejecta-CSM interaction. Explosion energies as high as $3 \times 10^{52}$ erg are required to reproduce the light curves 123 .

To denote these particularly energetic objects, the term "hypernova" 67 has been used, although its meaning is not well defined or universally accepted.

\section{$7 \quad$ Peculiar Supernovae}

Several objects do not fit the schema described above. In many cases they are probably core collapse SNe exploding in unusual configurations and/or conditions, even if the presence of other explosion mechanisms cannot be ruled out.

For instance, SN1993R [45] (and possibly SN1990aj, see, e.g., Fig. 44), showed hybrid spectral features of type Ia and Ib/c at late epochs. This has been interpreted as the explosion of a $\mathrm{SNIb} / \mathrm{c}$ with overproduction of ${ }^{56} \mathrm{Ni}$ or with the slow deflagration of a white dwarf.

The recent SN2000er was also classified as peculiar 3380. The scanty observations available show a broad maximum at $\mathrm{M}_{V}=-19$ followed by a rapid decline of 5 mag in 50 days. The spectrum shows (see, e.g., Fig. (4) a strong continuum with a drop at wavelengths shorter than $4000 \AA$, possibly due to the presence of metals. Narrow lines of He I with P-Cygni profiles are visible, indicating the presence of gas with expansion velocity $\sim 900 \mathrm{~km} \mathrm{~s}^{-1}$ as well as several other lines possibly due to FeII, FeIII and SiIII 21]. It might be a core collapse SN which lost its He envelope shortly before the explosion.

A well studied peculiar object is SN1961V, Zwicky's Type V, which was probably not a genuine SN because the exploding star survived the giant eruption 48. Similar objects might be SNe 1954J, 1999bw, and the faint SN1997bs for which an extremely luminous supergiant precursor has been identified [128].

\section{Conclusions}

The taxonomy of supernovae is a subject which is still evolving. The experience of the last years has shown that the diversity of SNe increases with our ability 
to detect, study, and understand them. Even though distant SNe are disclosing new frontiers in observational cosmology, much is still to be learned from nearby objects. Indeed, when we have the chance to apply new observational techniques or to obtain high signal-to-noise observations of nearby objects, new features and phenomena are revealed. Major progress in understanding supernovae can be expected if coordinated efforts involving several teams with wide ranging expertise and access to different observational facilities are established. But SN occurrences are unpredictable: in order to really understand them, we would need their cooperation.

\section{Acknowledgments}

This work is partially based on data collected at the European Southern Observatory (ESO) observatory on La Silla in Chile.

\section{References}

1. G. Aldering, R.M. Humphreys, M. Richmond: Astron. J. 107, 662 (1994)

2. I. Aretxaga, S. Benetti, R.J. Terlevich, A.C. Fabian, E. Cappellaro, M. Turatto, M. Della Valle: Mon. Not. R. Astron. Soc. 309, 343 (1999)

3. W.D. Arnett: Astrophys. J. 253, 785 (1982)

4. W.D. Arnett, J.N. Bahcall, R.P. Kirshner, S.E. Woosley: Ann. Rev. Astron. Astrophys. 27, 629 (1989)

5. W.D. Arnett, D. Branch, J.C. Wheeler: Nature 314, 337 (1985)

6. S. Balberg, L. Zampieri, S.L. Shapiro: Astrophys. J. 541, 860 (2000)

7. R. Barbon, S. Benetti, E. Cappellaro, F. Patat, M. Turatto, T. Iijima: Astron. Astrophys. Suppl. Ser. 110, 513 (1995)

8. R. Barbon, V. Buondi, E. Cappellaro, M. Turatto: Astron. Astrophys. Suppl. Ser. 139, 531 (1999)

9. R. Barbon, F. Ciatti, L. Rosino: Astron. Astrophys. 25, 241 (1973)

10. R. Barbon, F. Ciatti, L. Rosino: Astron. Astrophys. 72, 287 (1979)

11. N. Bartel et al.: Science 287, 112 (2000)

12. S. Benetti, E. Cappellaro, I.J. Danziger, M. Turatto, F. Patat, M. Della Valle: Mon. Not. R. Astron. Soc. 294, 448 (1998)

13. S. Benetti, F. Patat, M. Turatto, G. Contarini, R. Gratton, E. Cappellaro: Astron. Astrophys. 285, L13 (1994)

14. S. Benetti, M. Turatto, , P.A. Mazzali: Mon. Not. R. Astron. Soc. 305, 811 (1999)

15. S. Benetti et al.: Mon. Not. R. Astron. Soc. 322, 361 (2001)

16. F. Bertola: Annals Ap. 27, 319 (1964)

17. F. Bertola, A. Mammano, M. Perinotto: Contrib. Oss. Astrofis. Padova in Asiago 174, 51 (1965)

18. R.M. Bionta et al.: Phys. Rev. Lett. 58, 1494 (1987)

19. J.S. Bloom et al.: Nature 401, 453 (1999)

20. D. Branch, M. Livio, L.R. Yungelson, F.R. Boffi, E. Baron: Pub. Astron. Soc. Pacific 107, 1019 (1995)

21. D. Branch, R. Thomas: private communication (2001)

22. D. Branch et al.: Astrophys. J. 566, 1005 (2002) 
23. E. Cappellaro, I.J. Danziger, M. Turatto: Mon. Not. R. Astron. Soc. 277, $106(1995)$

24. E. Cappellaro, P. A. Mazzali, S. Benetti, I. J. Danziger, M. Turatto, M. Della Valle, F. Patat: Astron. Astrophys. 328, 203 (1997)

25. E. Cappellaro, M. Turatto: In: The Influence of Binaries on Stellar Population Studies, ed. by D. Vanbeveren (Kluwer Academic Publishers, Dordrect 2001) p. 199

26. E. Cappellaro, M. Turatto, P. A. Mazzali: IAUC 7091 (1999)

27. E. Cappellaro et al.: Astrophys. J. Lett. 549, L215 (2001)

28. R.A. Chevalier: Astrophys. J. 259, 302 (1982)

29. R.A. Chevalier: Astrophys. J. Lett. 259, L85 (1982)

30. R.A. Chevalier, C. Fransson: Astrophys. J. 420, 268 (1994)

31. N.N. Chugai: Sov. Astron. 12, L192 (1986)

32. N.N. Chugai, I.J. Danziger: Mon. Not. R. Astron. Soc. 268, 173 (1994)

33. A. Clocchiatti, M. Turatto: IAUC 7528 (2000)

34. A. Clocchiatti, J.C. Wheeler: Astrophys. J. 491, 375 (1997)

35. A. Clocchiatti, J.C. Wheeler, M.S. Brotherton, A.L. Cochran, D. Wills, E.S. Barker, M. Turatto: Astrophys. J. 462, 462 (1996)

36. A. Clocchiatti et al.: Astron. J. 111, 1286 (1996)

37. A. Clocchiatti et al.: Astrophys. J. 536, 62 (2000)

38. A. Clocchiatti et al.: Astrophys. J. 553, 886 (2001)

39. J.H. Elias, J.A. Frogel, J.A. Hackwell, S.E. Persson: Astrophys. J. Lett. 251, L13 (1981)

40. A. Fassia et al.: Mon. Not. R. Astron. Soc. 325, 907 (2001)

41. R.A. Fesen: Astrophys. J. Lett. 413, L109 (1993)

42. A.V. Filippenko: Astron. J. 96, 194 (1988)

43. A.V. Filippenko: Pub. Astron. Soc. Pacific 101, 588 (1989)

44. A.V. Filippenko: Ann. Rev. Astron. Astrophys. 35, 309 (1997)

45. A.V. Filippenko: In: Thermonuclear Supernovae, ed. by P. Ruiz-Lapuente, R. Canal, J. Isern (Kluwer Academic Publishers, Dordrect 1997) p. 795

46. A.V. Filippenko: IAUC 6969 (1998)

47. A.V. Filippenko, W.L.W. Sargent: Nature 316, 407 (1985)

48. A.V. Filippenko, A.J. Barth, G.C. Bower, L.C. Ho, G.S. Stringfellow, R.W. Goodrich, A. Porter: Astron. J. 110, 2261 (1995)

49. A.V. Filippenko et al.: Astrophys. J. Lett. 450, L11 (1995)

50. A. V. Filippenko et al.: Astron. J. 104, 1543 (1992)

51. D.W. Fox: Mon. Not. R. Astron. Soc. 319, 1154 (2000)

52. C. Fransson: Astron. Astrophys. 111, 140 (1982)

53. C. Fransson: Astron. Astrophys. 133, 264 (1984)

54. C. Fransson, P. Lundqvist, R.A. Chevalier: Astrophys. J. 461, 993 (1996)

55. T. J. Galama et al.: Nature 395, 670 (1998)

56. P. Garnavich, S. Jha, R.P. Kirshner, P. Berlind: IAUC 7066 (1998)

57. P. Garnavich, S. Jha, R.P. Kirshner, P. Challis, D. Balam, W. Brown, C. Briceno: IAUC 6786 (1997)

58. C.M. Gaskell, E. Cappellaro, H.L. Dinerstein, D. Garnett, R.P. Harkness, J.C. Wheeler: Astrophys. J. Lett. 306, L77 (1986)

59. L.M. Germany, D.J. Reiss, B.P. Schmidt, C.W. Stubbs, E.M. Sadler: Astrophys. J. 533, 320 (2000)

60. B.K. Gibson et al.: Astrophys. J. 529, 723 (2000)

61. M. Hamuy, S.C. Trager, P.A. Pinto, M.M. Phillips, R.A. Schommer, V. Ivanov, N.B. Suntzeff: Astron. J. 120, 1479 (2000) 
62. R.P. Harkness, J.C. Wheeler: In: Supernovae, ed. by A.G. Petshek (Springer, Berlin 1990) p. 1

63. R.P. Harkness et al.: Astrophys. J. 317, 355 (1987)

64. K. Hirata et al.: Phys. Rev. Lett. 58, 1490 (1987)

65. P. Höflich, J.C. Wheeler, L. Wang: Astrophys. J. 521, 179 (1999)

66. D.A. Howell: Astrophys. J. Lett. 554, L193 (2001)

67. K. Iwamoto et al.: Nature 395, 672 (1998)

68. S. Jha et al.: Astrophys. J. Suppl. 125, 73 (1999)

69. B. Leibundgut: Astron. Astrophys. Rev. 10, 179 (2000)

70. B. Leibundgut et al.: Astrophys. J. 372, 531 (1991)

71. B. Leibundgut et al.: Astron. J. 105, 301 (1993)

72. E.J. Lentz et al.: Astrophys. J. 507, 406 (2001)

73. D.C.; Leonard, A.V. Filippenko, A.J. Barth, T. Matheson: Astrophys. J. 536, $239(2000)$

74. P. Lira et al.: Astron. J. 116, 1006 (1998)

75. L.B. Lucy: Astrophys. J. 383, 308 (1991)

76. T. Matheson, A.V. Filippenko, R. Chornock, D.C. Leonard, W. Li: Astron. J. 119, 2303 (2000)

77. T. Matheson, A.V. Filippenko, L.C. Ho, A.J. Barth, D.C. Leonard: Astron. J. 120, 1499 (2000)

78. T. Matheson, A.V. Filippenko, W. Li, D.C. Leonard, J.C. Shields: Astron. J. 121, 1648 (2001)

79. T. Matheson et al.: Astron. J. 120, 1487 (2000)

80. A. Maury, I. Hook, R. Gorki, F. Selman, M. Dennefeld: IAUC 7528 (2001)

81. J. Maza, S. van den Bergh: Astrophys. J. 204, 519 (1976)

82. P.A. Mazzali, E. Cappellaro, I.J. Danziger, S. Benetti, M. Turatto: Astrophys. J. Lett. 499, L49 (1998)

83. P.A. Mazzali, K. Iwamoto, K. Nomoto: Astrophys. J. 545, 407 (2000)

84. P.A. Mazzali, K. Nomoto, F. Patat, K. Maeda: Astrophys. J. 559, 1047 (2001)

85. R.J. McMillan, R. Ciardullo: Astrophys. J. 473, 707 (1996)

86. W.P.S. Meikle: Mon. Not. R. Astron. Soc. 314, 782 (2000)

87. P.A. Milne, L.S. The, M.D. Leising: Astrophys. J. Suppl. 124, 503 (1999)

88. R. Minkowski: Mon. Not. R. Astron. Soc. 53, 224 (1941)

89. K. Nomoto, K. Iwamoto, T. Suzuki: Phys. Rep. 256, 173 (1995)

90. P. Nugent, M.M. Phillips, E. Baron, D. Branch, P. Hauschildt: Astrophys. J. Lett. 455, L147 (1995)

91. A. Pastorello et al.: Mon. Not. R. Astron. Soc. 333, 27 (2002)

92. F. Patat, R. Barbon, E. Cappellaro, M. Turatto: Astron. Astrophys. Suppl. Ser. 98, 443 (1993)

93. F. Patat, R. Barbon, E. Cappellaro, M. Turatto: Astron. Astrophys. 282, 731 (1994)

94. F. Patat, N. Chugai, P.A. Mazzali: Astron. Astrophys. 299, 715 (1995)

95. F. Patat, A. Piemonte: IAUC 6918 (1998)

96. F. Patat et al.: Astrophys. J. 555, 900 (2001)

97. S. Perlmutter et al.: Astrophys. J. 483, 565 (1997)

98. M.M. Phillips: Astrophys. J. Lett. 413, L105 (1993)

99. M.M. Phillips, S.R. Heatcote, M. Hamuy, M. Navarrete: Astron. J. 95, 1087 (1988)

100. M.M. Phillips, P. Lira, N.B. Suntzeff, R.A. Schommer, M. Hamuy, J. Maza: Astron. J. 118, 1766 (1999) 
101. A.C. Porter, A.V. Filippenko: Astron. J. 93, 1372 (1987)

102. Y.P. Pskovskii: Astron. Zhurnal 44, 82 (1967)

103. D.E. Reichart: Astrophys. J. Lett. 521, L111 (1999)

104. A.G. Riess et al.: Astron. J. 116, 1009 (1998)

105. P. Ruiz-Lapuente, E. Cappellaro, M. Turatto, C. Gouiffes, I.J. Danziger, M. della Valle, L.B. Lucy: Astrophys. J. Lett. 387, L33 (1992)

106. E.M. Sadler, R.A. Stathakis, B.J. Boyle, R.D. Ekers: IAUC 6901 (1998)

107. A. Saha, A. Sandage, G.A. Tammann, L. Labhardt, F.D. Macchetto, N. Panagia: Astrophys. J. 522, 802 (1999)

108. A. Saha et al.: Astrophys. J. 551, 973 (2001)

109. M. Salvo, E. Cappellaro, P.A. Mazzali, S. Benetti, I.J. Danziger, F. Patat, M. Turatto: Mon. Not. R. Astron. Soc. 321, 254 (2001)

110. E.M. Schlegel: Mon. Not. R. Astron. Soc. 224, 269 (1990)

111. B. Schmidt, R.P. Kirshner, B. Leibundgut, L. Wells, A.C. Porter, P. RuizLapuente, A.V. Filippenko: Astrophys. J. Lett. 434, L19 (1994)

112. B. Schmidt et al.: Astron. J. 107, 1444 (1994)

113. S.J. Smartt, G.F. Gilmore, A. Christopher, C.A. Tout, S.T. Hodgkin: Astrophys. J. 565, 1089 (2002)

114. S.J. Smartt, G.F. Gilmore, N. Trentham, C.A. Tout, C.M. Frayn: Astrophys. J. Lett. 556, L29 (2001)

115. N.B. Suntzeff: In: Supernova and Supernova Remnants, ed. by R. McCray, Z. Wang (Cambridge University Press, Cambridge 1996) p. 41

116. N.B. Suntzeff et al.: Astron. J. 117, 1175 (1999)

117. R.J. Terlevich, G. Tenorio-Tagle, J. Franco, J. Melnick: Mon. Not. R. Astron. Soc. 255, 713 (1992)

118. S.E. Thorsett, D.W. Hogg: GCN 197 (1999)

119. M. Turatto, S. Benetti, E. Cappellaro, I.J. Danziger, M. Della Valle, C. Gouiffes, P.A. Mazzali, F. Patat: Mon. Not. R. Astron. Soc. 283, 1 (1996)

120. M. Turatto, E. Cappellaro, I.J. Danziger, S. Benetti: Mon. Not. R. Astron. Soc. 265, 471 (1993)

121. M. Turatto, E. Cappellaro, I.J. Danziger, S. Benetti, C. Gouiffes, M. Della Valle: Mon. Not. R. Astron. Soc. 262, 128 (1993)

122. M. Turatto et al.: Astrophys. J. Lett. 498, L129 (1998)

123. M. Turatto et al.: Astrophys. J. Lett. 534, L57 (2000)

124. A. Uomoto, R.P. Kirshner: Astrophys. J. 308, 685 (1986)

125. S. van den Bergh, D. Branch: Astron. J. 105, 2231 (1993)

126. S. van den Bergh, J. Pazder: Astrophys. J. 390, 34 (1992)

127. S.D. Van Dyk, M. Hamuy, A.V. Filippenko: Astron. J. 111, 2017 (1996)

128. S.D. Van Dyk, C.Y. Peng, A.J. Barth, A.V. Filippenko: Astron. J. 118, 2331 (1999)

129. S.D. Van Dyk, K.W. Weiler, R.A. Sramek, M.P. Rupen, N. Panagia: Astrophys. J. Lett. 432, L115 (1994)

130. S.D. Van Dyk, K.W. Weiler, R.A. Sramek, E.M. Schlegel, A.V. Filippenko, N. Panagia, B. Leibundgut: Astron. J. 111, 1271 (1996)

131. L. Wang, J.C. Wheeler: Astrophys. J. Lett. 504, L87 (1998)

132. K.W. Weiler: http://rsd-www.nrl.navy.mil/7214/weiler/kwdata/RSNtable.txt

133. K.W. Weiler, N. Panagia, M.J. Montes: Astrophys. J. 562, 670 (2001)

134. J.C. Wheeler, S. Benetti: In: Allen's Astrophysical Quantities, ed. by A.N. Cox (Springer, New York 2000) p. 451

135. J.C. Wheeler, R.P. Harkness: Rep. Prog. Phys. 53, 1467 (1990) 
136. J.C. Wheeler, R.P. Harkness, E.S. Barker, A.L. Cochran, D. Wills: Astrophys. J. Lett. 313, L69 (1987)

137. J.C. Wheeler, R.P. Harkness, A. Clocchiatti, S. Benetti, M.S. Brotherton, D.L. DePoy, J. Elias: Astrophys. J. Lett. 436, L135 (1994)

138. J.C. Wheeler, R. Levreault: Astrophys. J. Lett. 294, L17 (1985)

139. S.E. Woosley, R.G. Eastman, B.P. Schmidt: Astrophys. J. 516, 788 (1999)

140. S.E. Woosley, T.A. Weaver: Ann. Rev. Astron. Astrophys. 24, 205 (1986)

141. Q. Yulei, W. Li, Q. Qiyuan, H. Jingyao: Astron. J. 117, 736 (1999)

142. H.U. Zimmerman et al.: Nature 367, 621 (1994)

143. F. Zwicky: In: Stars and Stellar Systems, ed. by L.H. Aller, D.B. McLaughin (University of Chicago Press, Chicago 1965) p. 367 